\title{
Design of Intelligent Detection Platform for Waterfowl Breeding Environment Based on Internet of Things
}

\author{
Ling Yang ${ }^{1}$, Xucan $\mathrm{Cai}^{2}$, Yun $\mathrm{Liu}^{3}$ \\ School of Information Science \& Technology, Zhongkai University of Agriculture and Engineering, \\ Guangzhou, China \\ 1yang98613@163.com , 2971577919@qq.com, ${ }^{3}$ lauwin007@163.com
}

\section{Keywords: IoT; RFID; ZigBee; Waterfowl; Environmental Detection.}

\begin{abstract}
The quality of breeding environment is one of the most important bottlenecks that restrict the sustainable development of waterfowl breeding. Good breeding environment can reduce the growth of disease or the harms brought by the disease, and improve the waterfowl's quality and quantity. This paper designs and implements a remote monitoring platform for waterfowl breeding environment based on internet of things. The platform is composed by three modules: remote monitoring/controlling terminal, front-end data processing center, and data acquisition terminal. The data acquisition terminal uses the processor LPC11C14, which is based on Cortex-M0 core; the front-end data processing center uses a tablet computer based on Cortex-A8 processor to do data analysis and management. As shown in the data acquisition test, the server and the mobile terminal all can stably receive the monitoring data of the waterfowl breeding environment.
\end{abstract}

\section{Introduction}

China is the waterfowl breeding center of the world, its products has an absolute advantage in the international market. Guangdong province is one of the largest provinces in waterfowl's in the production and consumption market, it has "the hometown of waterfowl" reputation. According to incomplete statistics, in 2014, the number of waterfowls of Guangdong province reached $3,000,000,000,3.5$ percent bigger than that in 2013. Both the production of meat and eggs of waterfowl are more than the national average. Waterfowl breeding environment is one of the important bottlenecks that restrict the sustainable development of waterfowl breeding. Good breeding environment can reduce the growth of disease, or the harms brought by the waterfowl disease, and improve the waterfowl's quality and quantity. This paper establish an intelligent detecting platform for waterfowl breeding environment to monitor the environmental factors such as light, ventilation, temperature, air, etc. Then use these data to compare with the best index parameters of environmental factors, so the platform can early-warning the breeding environment to ensure the security of waterfowl breeding environment. At the same time, these waterfowl breeding environment's data can be saved, and use for the quality and safety traceability platform.

\section{Architecture of Platform}

\section{A. Overall Design of Platform}

The overall structure of the platform for intelligent detection is shown in Fig. 1. The platform use the sensor acquisition modules deployed in the goose house to get the information of environment, Cortex-M0 monitor module control the corresponding equipment by the ZigBee wireless network to timing acquire the environment information received by sensors, then send the information to the data processing center. The data processing center analyze the data and send the command of management platform to the monitor module. At the same time, data processing center can communicate with platform terminal (PC terminal, mobile terminal) by WiFi, and upload the data to the Web server. The user can access the Web server to remote monitor the breeding environment.

1 This work was supported in part by Natural Science Foundation of Guangdong Province (9151022501000008, 2 015A030313602), NSFC(61501531), Sci. \& Tech. project of Guangdong PR (2010A020507001-80), and Univers ity Outstanding Young Creative Talents Cultivation Project of Guangdong PR (LYM10089). 


\section{B. System Architecture}

The platform is mainly divided into three modules: remote monitoring control terminal, front-end data processing center and data acquisition terminal. The level structure of platform is shown in Fig.2.

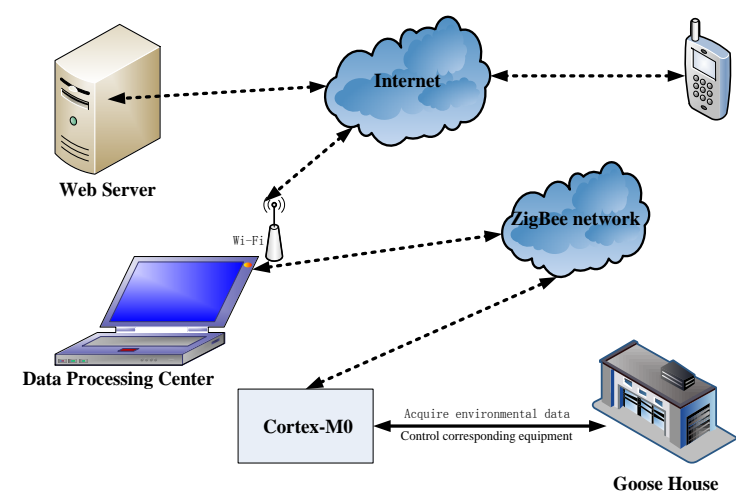

Fig. 1 Overall structure diagram of the platform

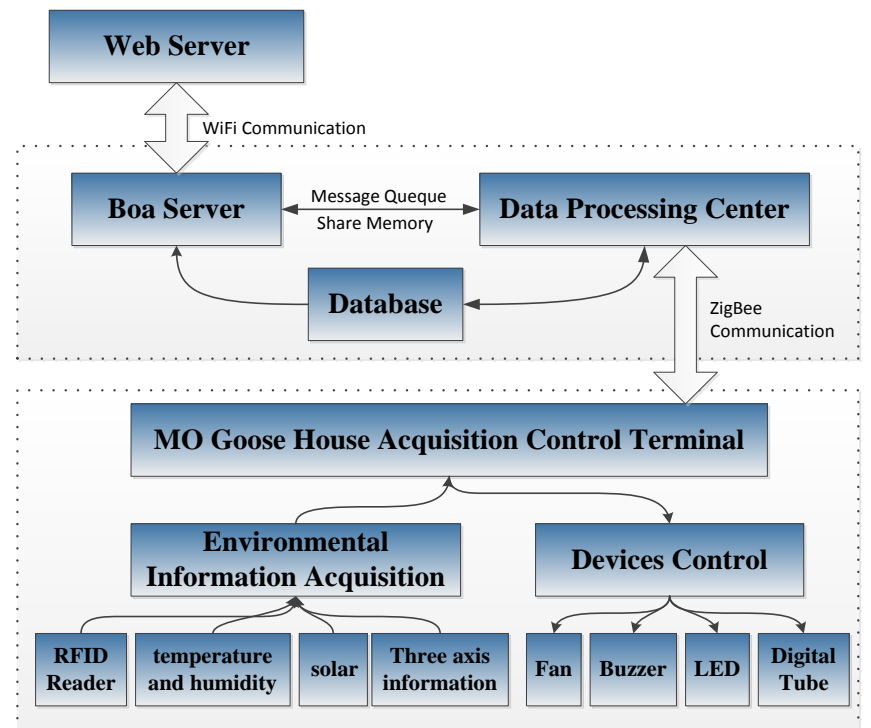

Fig. 2 Platform level structure diagram

\section{Design of data acquisition terminal}

\section{A. Design scheme of Cortex-M0 terminal}

The terminal design scheme is shown in fig. 3. From the figure, we can see the data acquisition terminal consist of data acquisition module (temperature and humidity sensor, solar sensor, RFID reader, etc.), ZigBee communication module, and data feedback terminal (LED, buzzer, fun, digital tube, etc.). Data acquisition module is responsible for collecting environmental data such as temperature and humidity, illumination. After the preliminary data processing, ZigBee communication module sends the data to A8 data processing center. At the same time, ZigBee communication module receives the command from $A 8$ data processing center; control the data feedback terminal for real-time operation.

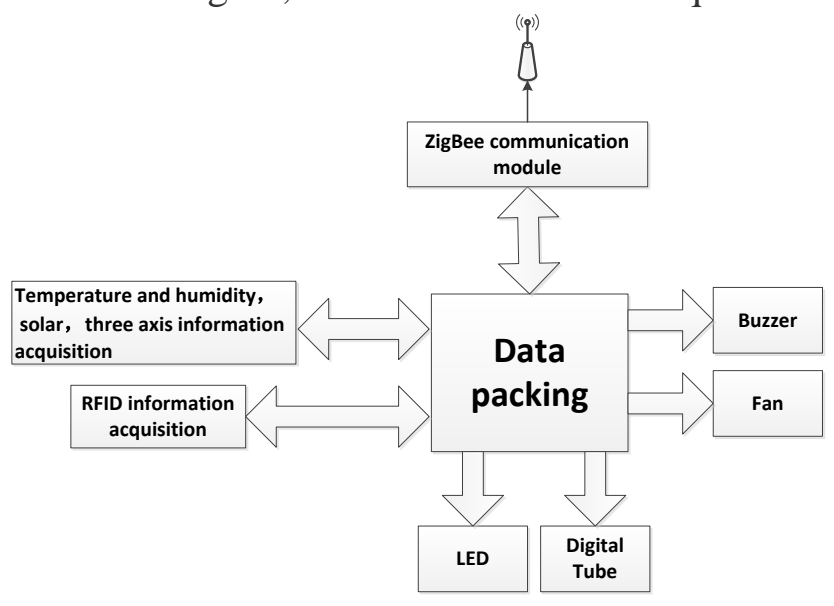

Fig. 3 Cortex-M0 terminal design scheme

ZigBee wireless communication has the advantages of low power consumption, low cost, Ad-Hoc Network, high reliability, automatic network restoration, excellent redundancy; multiple ZigBee nodes can form a wireless communication network, etc. The scheme uses the ZigBee communication module to be responsible for the communication between $\mathrm{M} 0$ data acquisition terminal and A8 data processing center, the communication between the data acquisition terminal and all the sensors. The interface between Zigbee and data acquisition terminal use serial peripheral interface (SPI) bus, UART to USB (PL2303HX) chip.

\section{B. Workflow of Cortex-M0 terminal}

The workflow of Cortex-M0 terminal is shown in fig.4. 


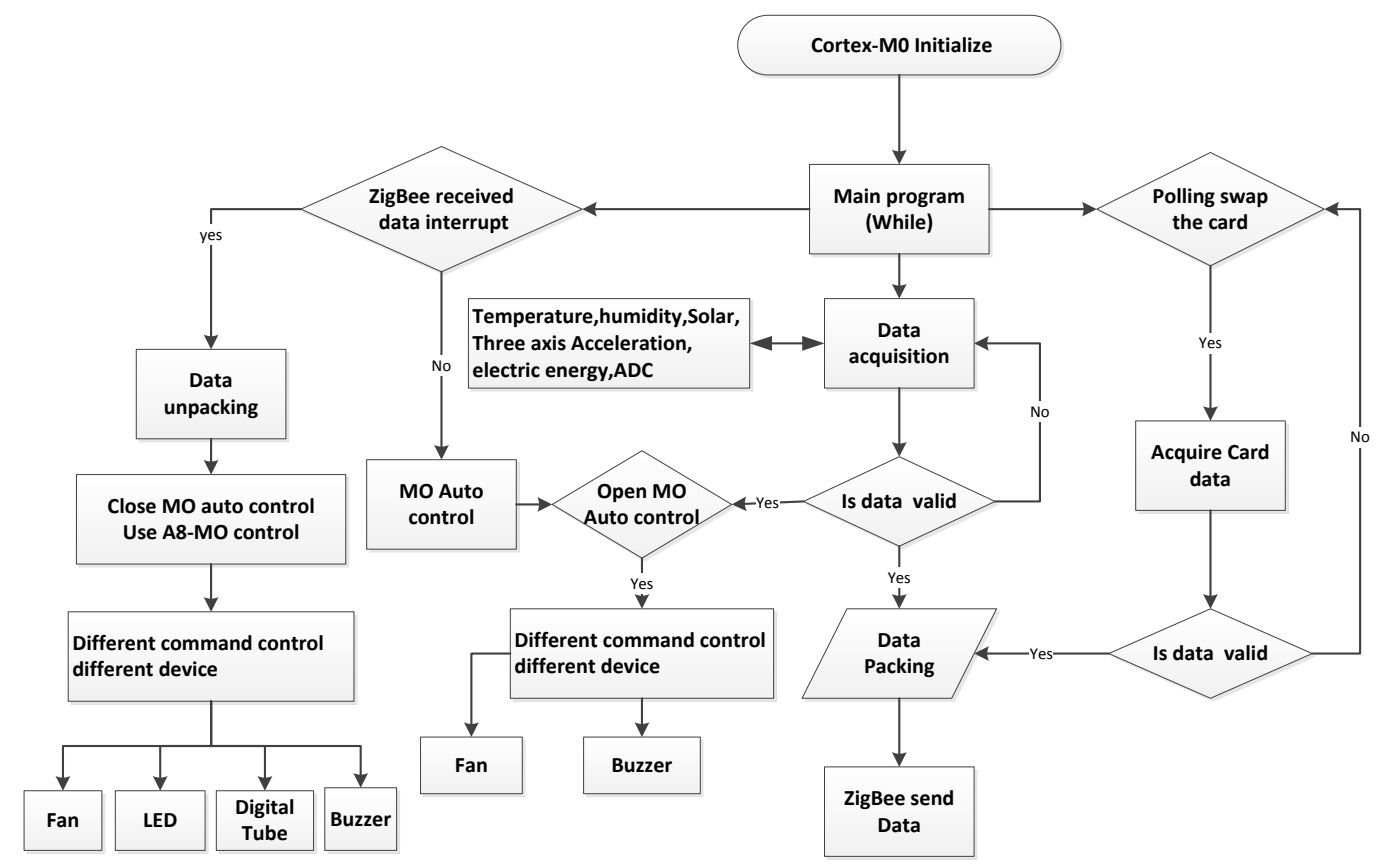

Fig. 4 Main workflow of Cortex-M0 terminal

a. Initialize all the equipment of data acquisition terminal (timer, SPI, UART, etc). Among them, set the timer for 1 milliseconds, set the interrupt interval of interrupt function for 1 second.

b. when the system does not detect the interrupt (there is two interrupts: one happened when RFID reader swiped the card, the other happened when the ZigBee received the command from A8 data processing center), it is mainly carried out environmental data acquisition. Then it will judge the data is valid or not, if the data is valid it will pack the data and send it to the A8 data processing center. At the same time, when the system does not receive the control command, it will automatic control the fan, buzzer, display to send out an alarm to show abnormal.

c. when the system detect the RFID swipe card interrupt, RFID interrupt function will acquire the data from RFID reader. Then check the data, if it is valid the system will send it to the A8 data processing center.

d. when the system detect the ZigBee received data interrupt, ZigBee received data interrupt function will receive the command. Unpack the data and check the data, then control the device such as fan, buzzer, LED etc, to carry out the command. At the same time, it will shut down the MO control mode, switch to A8 control mode.

e. To ensure the system can run stalely, ensure the system can run normal under abnormal situation, we will set the system will automatic switch to MO control mode when the data acquisition terminal does not receive the command from A8 data processing center in 30 second. Sent abnormal warning or not will be judged by MO data acquisition terminal.

\section{Temperature and Humidity Sensor DHT11}

DHT11 is a compound sensor of temperature and humidity. It use single bus to communicate, consist of 8 bits single chip microcomputer, humidity sensor and temperature sensor. Every time can send 40 bit data by high bits out first and low bits out last, the format of data is shown in table 1 .

Table 1 Format of data bit

\begin{tabular}{cccccc}
\hline data bit & $39-32$ & $31-24$ & $23-16$ & $15-8$ & $7-0$ \\
\hline meaning & $\begin{array}{l}\text { Temperature } \\
\text { integer part }\end{array}$ & $\begin{array}{l}\text { Temperature } \\
\text { decimal part }\end{array}$ & $\begin{array}{l}\text { Humidity integer } \\
\text { part }\end{array}$ & $\begin{array}{l}\text { Humidity decimal } \\
\text { part }\end{array}$ & Check bit \\
\hline
\end{tabular}

The principle of DHT11 temperature and humidity acquisition module is shown in fig. 5. The data output port of temperature sensor is connected to the PIO1_5 pin of the I/O port on the LPC_11C14. It use single bus control mode to read the temperature value. The workflow of DHT11 is shown in fig.6. 


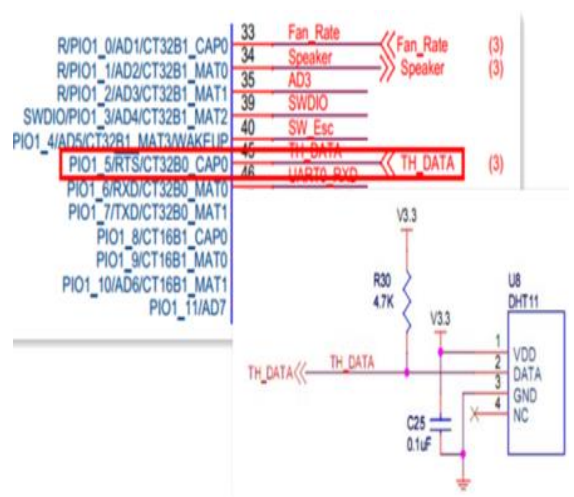

Fig. 5 Principle of DHT11

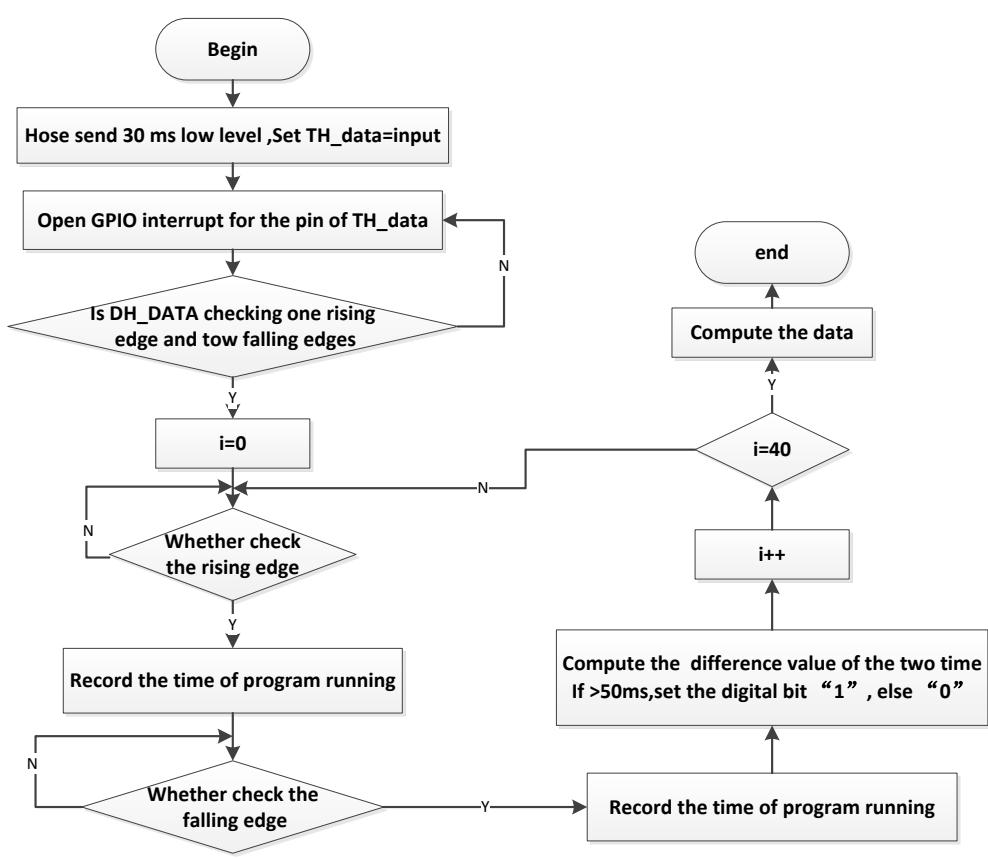

Fig. 6 Workflow of DHT11

\section{Solar Sensor ISL29003}

Solar sensor ISL29003 is integrated optical sensors of 16-bit ADC type. It has built-in ADC interface and IIC interface. The ADC provide 16 effective decomposition, the light intensity it acquired is between 1 to 100,000 lux. The data output is digital output, it has more noise immunity than analog output, more easily transmission by I2C bus protocol. ISL29003 uses $\mathrm{I} 2 \mathrm{C}$ bus to communicate (principle is shown in figure 7). I2C bus has serial clock line (SCL) and serial date Line (SDA). When the

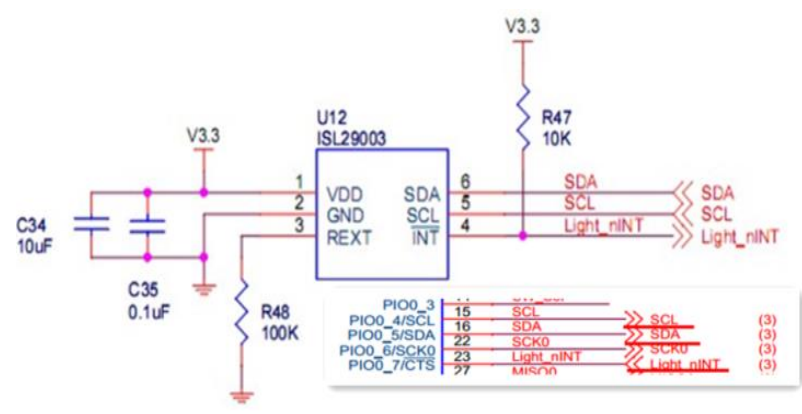

Fig. 7 Principle of ISL29003 module

SDA is stable at high level, the sensor can transmit data, and the data can not be transmitted at low level. The level state of SDA is controlled by SCL, when SCL is at low level, the level state of SDA is able to change; when SCL is at high level, the level state of SDA is not able to change.

\section{E. RFID Reader CY14443}

CY14443A RFID reader use special chip of non-contact card reader based on ISO14443 standard, use SPI bus protocol to communicate. SPI is a synchronization protocol interface, all the transmission is reference to the same clock signal, the synchronization clock signal is generated by the host. SPI main has 4 signals: host output/ slave output, host input/ slave input, serial clock (SCK) and peripheral chip select (CS). The collection model is shown in fig. 8 .

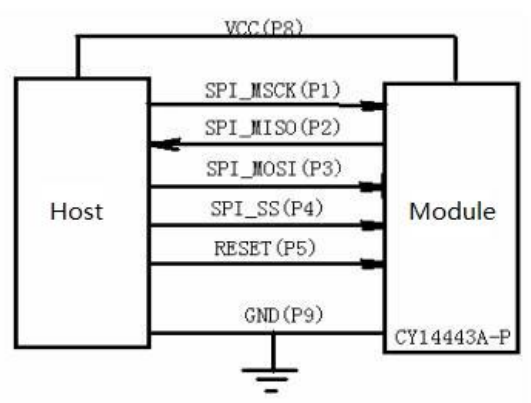

Fig. 8 Collection model of SPI

\section{F. ZigBee Wireless Communication}

ZigBee wireless communication module use CC2530 chip of TI Company, CC2530 integrate enhanced $8051 \mathrm{CPU}$, can program online. CC2530 integrate the chip that has function of USB interface to serial interface, so it can easily communicate with USB device, can network with FS_11C14 to communicate. The kernel chip ZIC2410 comply with ZigBee specification and IEEE802.15.4 standard. ZIC2410 is consist of RF transceiver with the baseband modem, hardwired MAC and microcontroller with 8051 kernel(with an internal Flash memory). 
Communication protocol adopts master-slave asynchronous communication method, use the A8 data processing center as master machine, MO data receive terminal as slave machine. Before communication needs to build links, it means that the master machine will send a request signal

"Y" to the slave first, when the slave machine receive the signal it will send a responsive signal

"A" ; when the master machine receive the responsive signal, the communication links will be built. Master slave data transmission adopt poll mode, that means slave machine send a data to the master machine per second.

\section{Design of Data Processing Center}

\section{A. Overall Design of Data Processing Center Based on Cortex-A8}

Cortex-A8 data processing center uses multi-thread programming technology development, M0 data receiving thread receive the environmental information and the goods information, activate the database thread to do the corresponding processing to the database, refresh the shared memory, and then to compare the environmental information with the parameters of environmental information, after judgment, it will process the environmental information or send an alert. Web monitoring terminal CGI process and A8 use the message queue to communicate, through the M0 data control thread to achieve web monitoring. The relationship between threads is shown in fig. 9.

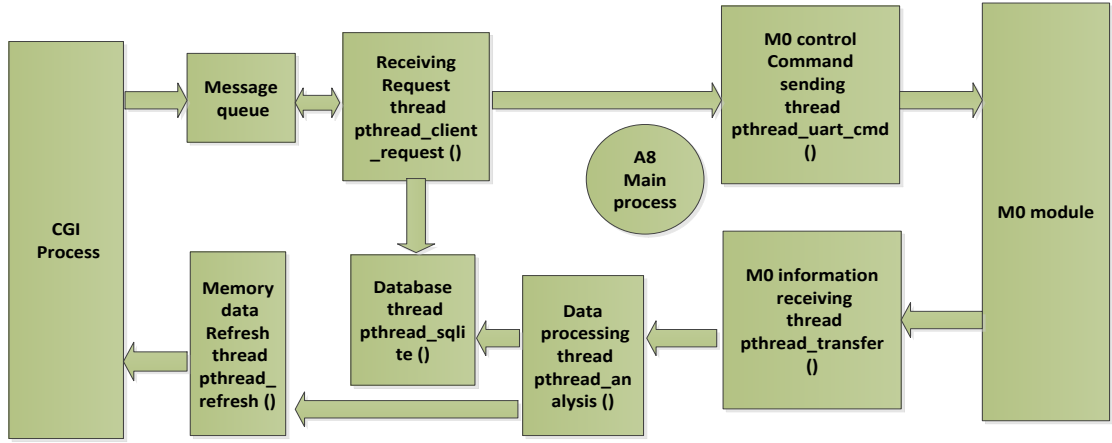

Fig. 9 Relationship Between Threads

\section{B. Design of Data Receiving Module}

Data receiving module is responsible for receiving data from the data acquisition terminal, Data is transmitted to the serial port through the USB interface, and the data read is processed by the data processing module. Program flow chart is shown in Fig. 10.

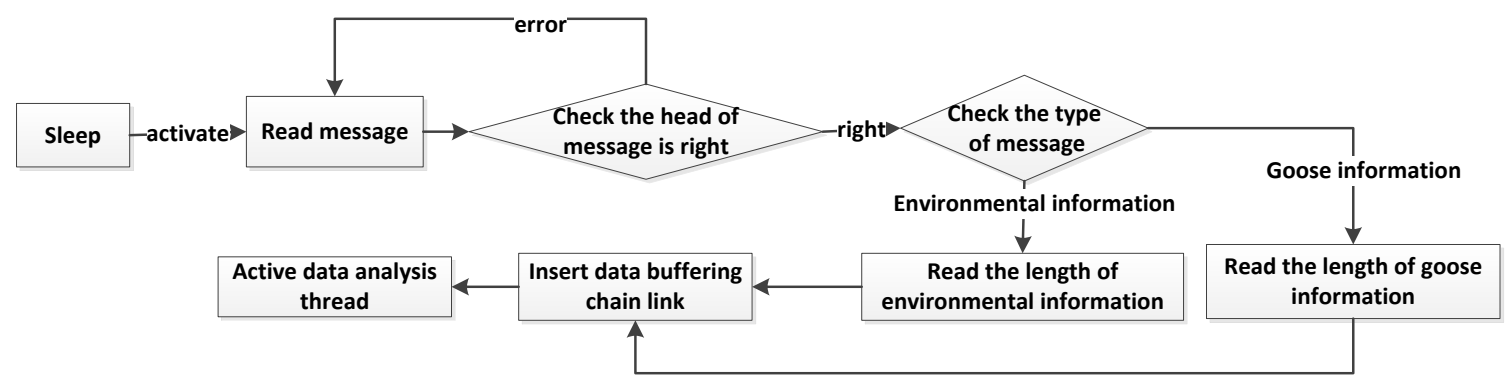

Fig. 10 Flow of data receiving thread

\section{Design of Data Analysis and Processing Module}

Because the received information is encoded according to a certain rule, so the data received needs to be decoded. After decoding analysis, it will activate the database thread to save the data, activate memory to refresh the data, update the real-time environmental information, and judge whether the data is out of the border, if the data is out of border, it will activate the device control thread to do the corresponding control. The flow of data analysis and processing thread is shown in Fig. 11. 


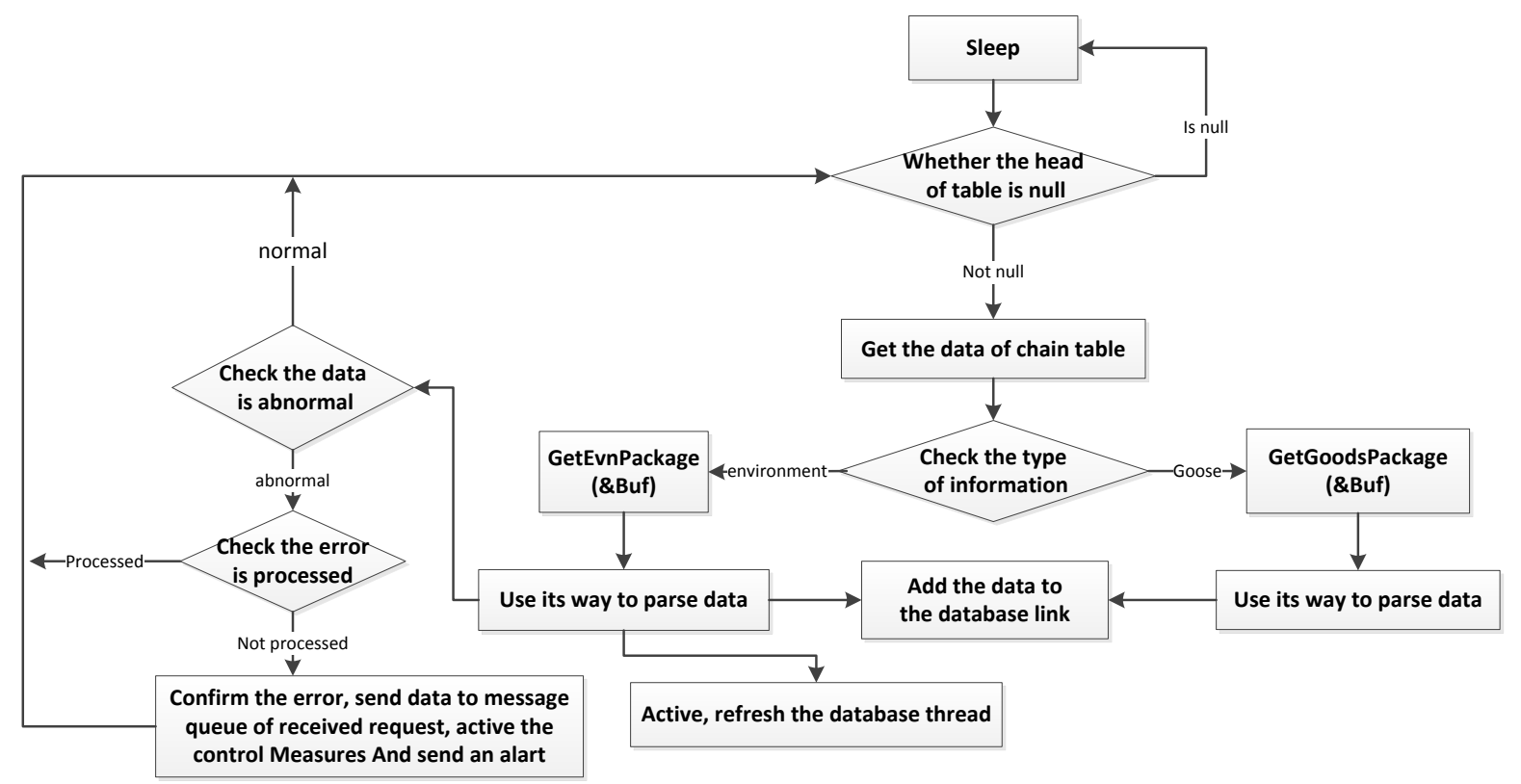

\section{Function Test}

Fig. 11 Flow of data analysis and processing thread

\section{A. Independent Function Test of Data Acquisition Terminal}

Under the normal communication between MO and A8, disconnect the connection between ZigBee wireless communication and A8 and MO, that is to switch to automatic control mode, then the MO can check whether its own environment is abnormal and alarm. At this moment, the display of LED will show "environment safe!" and the environmental data. When the temperature and humidity is out of the border, the position of environment will show "FAN 500" , that means the fan will begin to rotate at a speed of $500 \mathrm{rpm}$, at the same time the buzzer will ring.

\section{B. Connecting M0 and A8 Tablet for Integration Test}

Integration test use putty software to carry out system connection test. Connecting A8 tablet and PC by USB data cable, and do corresponding setting and monitor in putty. At the same time start the go script after the connection between ZigBee terminals and tablet, waiting for a response by MO. The Effect is shown in Fig. 12.

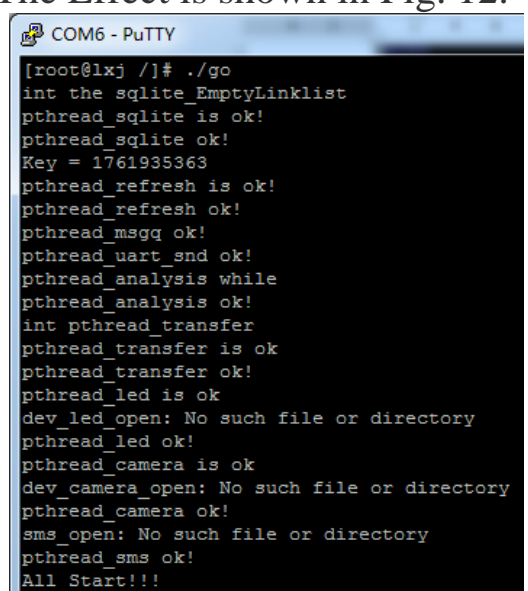

Fig. 12 Effect of integration test

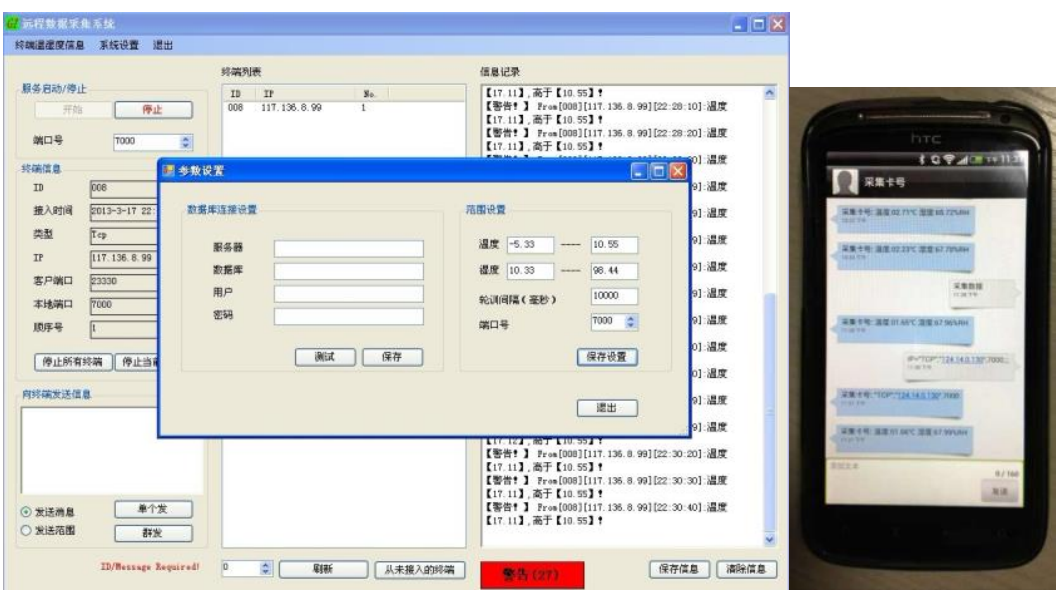

Fig. 13 Effect of MO show environment data

After the startup of MO, achieve the connection between A8 and M0, at this moment, the display of M0 will show the environmental data acquired, the effect is shown in fig. 13.

\section{Conclusions}

According to the characteristics of high temperature and humidity in Guangdong, this paper research and develops a remote monitoring platform for waterfowl breeding environment based on internet of things. The platform is mainly divided into three modules: remote monitoring control terminal, front-end data processing center and data acquisition terminal. The remote monitoring 
control terminal adopts boa server, uses CGI and HTML to show information. The front-end data processing center is developed by Cortex-A8 processor, uses Sqlte3 database to save data, and interacts information with CGI program process. The data acquisition terminal is developed by Cortex-M0 microprocessor, through the Zigbee network to communicate with other devices such as temperature and humidity sensor DHT11, solar sensor ISL29003, MMA7455L, RFID reader CY14443, etc; complete the data acquisition and the data interaction. After the test of unit test for data acquisition and integration test, server and mobile terminal all can steady receive the environmental monitoring data.

\section{Reference}

[1] Yang Wei, Li Minzan, Wang Xiu. Status quo and progress of data transmission and communication technology in field information acquisition[J]. Transactions of the Chinese Society of Agricultural Engineering, 2008, 24(5):297-301.

[2] Han Huafeng, Du Keming, Sun Zhongfu,et al. Design and application of ZigBee based telemonitoring system for greenhouse environment data acquisition[J]. Transactions of the Chinese Society of Agricultural Engineering, 2009, 25(7):158 - 163.

[3] Bao Changchun, Shi Ruizhen, Ma Yuquan, et al. Design and realization of measuring and controlling system based on Zigbee technology in agricultural facilities[J].Transactions of the Chinese Society of Agricultural Engineering, 2007, 23(8):160-164.

[4] Zhang J, Li W, Han N, et al. Forest fire detection systembased on a ZigBee wireless sensor network[J]. Frontiers of Forestry in China, 2008, 3(3): 369.

[5] Zhang Ruirui, Zhao Chunjiang, Chen Liping, et al.Design of wireless sensor network node for field information acquisition[J]. Transactions of the Chinese Society of Agricultural Engineering, 2009, 25(11): $213-218$.

[6] Chen Yifei, Du Shangfeng. Consideration for some problems about large system control in intelligent agriculture(IA)[J]. Agricultural Engineering, 2011, 1(1):8-13.

[7] Hui-jian $\mathrm{Xu}$. The design and implemention of intelligent warehouse system based

on RFID technique, Manufacturing Automation, 2012,07:139-141.

[8] Zhiping Xu. Design and Achievement of Intelligent Warehouse warehouse Management System Based on ZigBee Technology[D]. Harbin Engineering University, 2012. 